\title{
High Gain Microstrip Antenna with Left-Handed Materials
}

\author{
Mao Xiaoqun \\ Chongqing College of Electronic Engineering, Chongqing, 401331, China \\ 187185482@qq·com
}

\begin{abstract}
This paper presents design and analysis of a novel S-shaped left-handed material based on the equivalent circuit theory. The effective permittivity and effective permeability of the novel S-shaped left-handed material are both negative in the range from $5 \mathrm{GHz}$ to $7 \mathrm{GHz}$. A conventional microstrip antenna is designed following the empirical formula, and a high gain microstrip antenna is designed with use of the novel S-shaped left-handed materials. The performances of the conventional antenna and the modified antenna are simulated by HFSS software. The simulation results show that the gain of the antenna increased by $3.94 \mathrm{~dB}$, and the half power beam width decreased by $38.9^{\circ}$. Therefore, it is concluded that the performance of microstrip antenna can be improved by using left-handed materials.
\end{abstract}

Keywords: left-handed material, microstrip antenna, gain, half power beam width

\section{Introduction}

In 1968, Veselago theoretically proposed the possibility of materials with different characteristics from conventional material. As they do not exist in nature, they are termed Metamaterials. Until 2000, this subject left neglected for absence of natural materials with these abnormal properties. The interest on Veselago's work was renewed since Pendry et $a l$. showed a two-dimensional array which exhibit negative refractive index [1]. Almost at the same time, Shelby et. al., used split-ring resonators (SRRs) and wires to obtain negative permittivity and permeability simultaneously. When the electromagnetic wave propagating in the traditional medium i.e., the materials with positive permittivity and permeability, the direction of the electric field $E$, the magnetic density $H$, and the direction of the propagation vector $k$ formed "right-handed"( $\mathrm{RH})$ rule based on the individual Maxwell curl equation of the electric field [2]. When the permittivity and permeability are negative, the vectors $E, H$, and $k$ forming a "left-handed" rule. Therefore, such materials are referred to "left-handed material" (LHM) or "negative index material", (NIM). In some literature, also known as "double negative material"(DNG) [3].Many attractive characteristics of left-handed material have been found such as the reversal of Snell's law, reversed Doppler effect, and Vavilov-Cerenkov effect $[1,4]$ etc. Because of these unique electromagnetic properties, left-handed material can be used for designing different microwave devices such as antenna [5], radome [6] and filters [7] etc [8-10].

The microstrip antennas are widely used in modern communication systems for their advantages such as low weight, low profile, low cost, and simple fabrication [11-12].The tendency of future communication development is globalization communication. This indicates that antennas should satisfy the requirement of wideband operations and high gain characteristics. Recently, the performances of the microstrip antenna with left-handed material were investigated [13]. Seven same left-handed material structures were arranged as a planar radome to increase the gain of the antenna, and the gain of the antenna was enhanced with a growing of $2.5 \mathrm{~dB}$, but the double-negative bandwidth of left-handed material is narrow [14]. Results show that the antenna system with 
left-handed material was more directive and has higher gain while the bandwidth improved slightly [15]. It is no doubt that how to further improve the bandwidth and radiation characteristics is still the challenge [16].

This paper is organized as follows. Section II proposes a novel S-shaped left-handed material based on equivalent circuit theory. Section III presents the performance of a fundamental microstrip antenna. As a comparison, a high gain microstrip patch antenna with left-handed material is designed in Section IV, and the performance of the modified antenna is also presented in this section. Finally, Section V draws a conclusion that the performance of the microstrip antenna can be improved by using left-handed material.

\section{Left-Handed Material Unit Cell Structure}

S-shaped resonator (SSR) was proposed in 2004 [17], shown in Figure 1 (a).In SSR, the frequency domain with negative permittivity and negative permeability are overlapped, hence the metallic rods are not required to make it left-handed material [18]. The S-shaped metallic branches are printed on opposite of the substrate. Viewing from top of the structure, an eightlike pattern is comprised of two S-shaped strips. Structural configuration is presented in Figure 1 (b). The value of each parameter is $a=0.28 \mathrm{~mm}$, $b=5.3 \mathrm{~mm}, c=1.6 \mathrm{~mm}, \quad d=0.6 \mathrm{~mm}, t=0.2 \mathrm{~mm}$, and $\varepsilon_{r}$ is 4.4 .

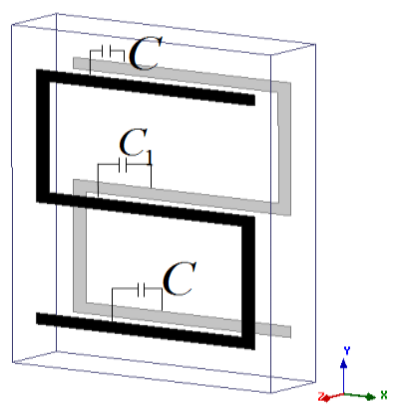

$1(\mathrm{a})$

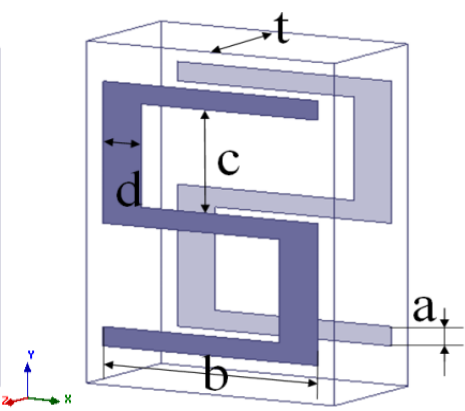

1 (b)

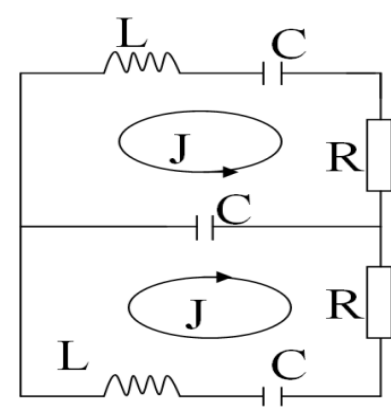

1 (c)

Figure 1. The Schematic of the S-shaped Left-Handed Material (a) Schematic Diagram of S-shaped Left-Handed Material; (b) Structural Configuration; (c) Equivalent Circuit

The boundary conditions are set as perfect electric condition (PEC) on $\mathrm{x}$-axis and perfect magnetic condition (PMC) on z-axis, wave ports are constructed in y direction. When the electromagnetic wave propagating through the S-shaped material, there are three capacitances among the circuit. The top metallic strips, center metallic strips, and bottom metallic strips formed capacitance $C$ respectively. Due to the presence of $C_{1}$, as showed in Figure 1 (a), the eightlike current loop is formed. Besides, the inductance is proportional to the area of loop. The equivalent inductance is represented [17] as

$$
L=\mu_{0} c(b-2 d)
$$

The equivalent capacitance [17] is

$$
C=\frac{\varepsilon_{0} \varepsilon_{r} a b}{t}
$$

The resonance frequency of the S-shaped material is [17]

$$
\omega_{m 0}=\sqrt{\frac{1}{L_{t} \sqsubset C_{t}}}=\sqrt{\frac{3 t}{\mu_{0} \varepsilon_{0} \varepsilon_{r} c a b(b-2 d)}}
$$


$L_{t}$ and $C_{t}$ denote the total inductance and the total capacitance, respectively. The plasma frequency of the S-shaped material is [17]

$$
\omega_{m p}=\frac{1}{\sqrt{1-F}} \omega_{m 0}
$$

$F$ is the fractional area of the unit cell [19], and the $F$ is calculated by

$$
F=\frac{2(b-2 d) c}{b(3 a+2 c)}
$$

Within the domain $\omega_{m 0}<\omega<\omega_{m p}$ the equivalent permittivity and permeability are negative.

According to the theory of equivalent circuit, the greater area ration value i.e. $F$, the greater coupling between the units, and the greater double negative bandwidth of the structure [20-21]. The horizontal-shift is introduced to the S-shaped material in this paper. Parameter $X$ is defined to represent the value of the horizontal-shift, shown in Figure 2 (a), and the equivalent circuit is presented in Figure 2 (b)

Due to the horizontal offset, the equivalent circuit is changed. As offset $X$ increasing the two inductances are not the same any more. One equivalent inductance increase, while the other decrease, $L_{1}$ and $L_{2}$ represent two different inductances as shown in Figure 2 (b). The equivalent capacitance change to be

$$
C=\frac{\varepsilon_{0} \varepsilon_{r} a(b-X)}{t}
$$

Thus, the total capacitance $C_{t}$ is

$$
C_{t}=\frac{2}{3} C
$$

And the two equivalent inductances represented are

$$
\begin{aligned}
& L_{1}=\mu_{0} c(b-X-2 d) \\
& L_{2}=\mu_{0} c(b+X-2 d)
\end{aligned}
$$

Thus, the total inductance $L_{t}$ is

$$
L_{t}=\frac{\mu_{0} c\left[(b-2 d)^{2}-X^{2}\right]}{2 b-4 d}
$$

The resonance frequency $\omega_{m 0}$ change to be

$$
\omega_{m 0}=\sqrt{\frac{1}{L_{t} \sqsubset C_{t}}}=\sqrt{\frac{3(b-2 d) t}{\mu_{0} \varepsilon_{0} \varepsilon_{r} c a(b-X)\left[(b-2 d)^{2}-X^{2}\right]}}
$$

the $F$ is calculated by

$$
F=\frac{(b+X-2 d) c+(b-X-2 d) c}{(b+X)(3 a+2 c)}
$$




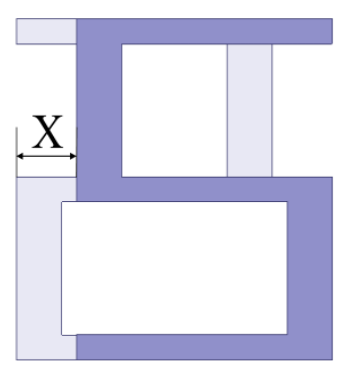

2(a)

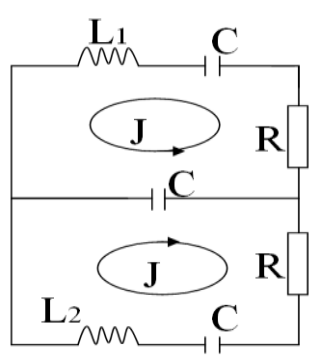

2(b)

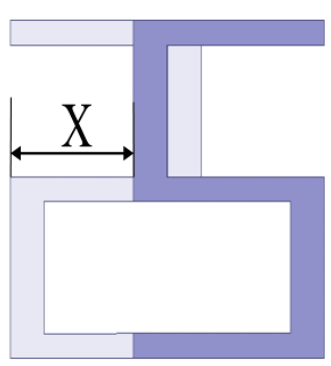

2(c)

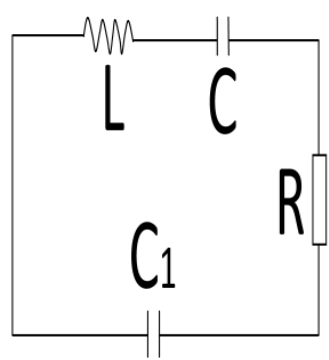

2(d)

Figure 2. The Schematic of the Novel S-Shaped (a) Schematic Diagram of Horizontal Offset; (b) Equivalent Circuit; (c) Schematic Diagram of Horizontal Offset; (d) Equivalent Circuit

When $X$ increases to only one loop left in the structure, as shown in Figure 2 (c). There are two capacitances in this equivalent circuit, they are respectively represented as

$$
\begin{gathered}
C=\frac{\left.2 a \varepsilon_{0} \varepsilon_{r}(b-X)\right)}{t}+\frac{\varepsilon_{0} \varepsilon_{r} c[(b-X)-2(b-X-d)]}{t} \\
C_{1}=\frac{\varepsilon_{0} \varepsilon_{r} a(b-j)}{t}
\end{gathered}
$$

The total capacitance $C_{t}$ change to be

$$
C_{t}=\frac{C_{1} C}{C_{1}+C}
$$

And the equivalent inductance is

$$
L=\mu_{0} c(b+j-2 d)
$$

In this case, the $F$ change to be

$$
F=\frac{(b+X-2 d) c}{(b+X)(3 a+2 c)-2 c X}
$$

And the $\omega_{m 0}$ is calculated by

$$
\omega_{m 0}=\sqrt{\frac{1}{C_{t} \square L}}
$$

The $\omega_{m p}$ is calculated by

$$
\omega_{m p}=\frac{1}{\sqrt{1-F}} \omega_{m 0}
$$

Based on the forementioned analysis, when $X=b-d$, the novel S-shaped material has wider bandwidth than the conventional S-shaped material.

The reflection coefficient $S_{11}$ and the transmission coefficient $S_{21}$ of this novel S-shaped material are calculated by the commercial software HFSS. The NRW retrieval procedure[19] is applied to calculate the equivalent permittivity and equivalent permeability from the scattering coefficient. 


$$
\begin{gathered}
n=\frac{1}{k t} \cos ^{-1}\left[\frac{1}{2 S_{21}}\left(1-S_{11}^{2}+S_{21}^{2}\right)\right] \\
z=\sqrt{\frac{\left(1+S_{11}\right)^{2}-S_{21}^{2}}{\left(1-S_{11}\right)^{2}-S_{21}^{2}}} \\
\varepsilon=n / z, \mu=n z
\end{gathered}
$$

Where $n$ is the refractive index and $z$ is the wave impedance, $k$ is the wave number of free space, $t$ is the thickness of the left-handed material structure.

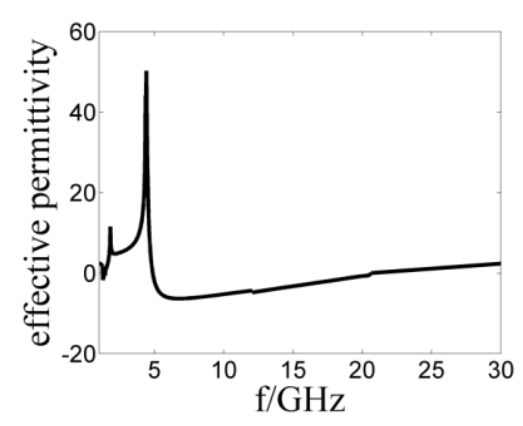

3(a)

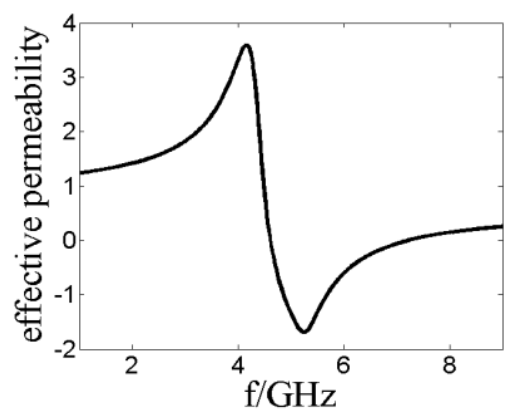

3(b)

\section{Figure 3. The Retrieved Parameter of the Novel S-shaped Left-Handed Material (a) Effective Permittivity, (b) Effective Permeability}

Figure 3. (a) gives the retrieved real parts of effective permittivity and effective permeability. It can be seen that in $5 \mathrm{GHz}-20 \mathrm{GHz}$, effective permittivity is negative, while the effective permeability is negative in $4.6 \mathrm{GHz}-7 \mathrm{GHz}$. Therefore, in the frequency ranges from $5 \mathrm{GHz}$ to $7 \mathrm{GHz}$, the effective permittivity and effective permeability are both negative.

\section{Microstrip Antenna}

The parameters of the microstrip antenna are calculated from the following formulas [11]. The width of the microstrip antenna is

$$
W=\frac{c}{2 f_{r}} \sqrt{\frac{2}{\varepsilon_{r}+1}}
$$

Where the $c$ represents for the free space velocity of light, the $f_{r}$ represents for the operating frequency, and the $\varepsilon_{r}$ represents for the dielectric constant of substrate.

The actual length of the microstrip antenna is

$$
L=L_{\text {eff }}-2 \Delta L
$$

Where the $L_{e f f}$ represents for the effective length of the patch, and $\Delta L$ is calculated by

$$
\Delta L=0.412 h \frac{\left(\varepsilon_{e}+0.3\right)(W / h+0.264)}{\left(\varepsilon_{e}-0.258\right)\left(W / h^{+0.8)}\right.}
$$


The $\varepsilon_{e}$ represents for the effective dielectric constant, and the $h$ represents for the height of the substrate.

The $L_{\text {eff }}$ is calculated by

$$
L_{\text {eff }}=\lambda_{g} / 2
$$

And the $\lambda_{g}$ represents for the wavelength of the material. The $\lambda_{g}$ is defined as

$$
\lambda_{g}=\lambda_{0} / \sqrt{\varepsilon_{e}}
$$

Where the $\lambda_{0}$ is represents for the free space wavelength. The $\varepsilon_{e}$ is calculated by

$$
\varepsilon_{e}=\frac{\varepsilon_{r}+1}{2}+\frac{\varepsilon_{r}-1}{2}\left(1+12 \frac{h}{W}\right)^{-\frac{1}{2}}
$$

The antenna configuration is shown in Figure 4 (a) and the size of antenna is $36 \times 32 \mathrm{~mm}^{2}$. The size of the patch is $11.2 \times 5.8 \mathrm{~mm}^{2}$. The microstrip antenna is designed on FR-4 substrate and the height of the substrate is $2 \mathrm{~mm}$. The inner conductor of the coaxial feed passes through a hole and electrically connected to the patch. The HFSS software is used to analyze the microstrip patch antenna.

Simulated result of return loss is shown in Figure 4 (c). It is indicated the bandwidth of microstrip antenna is $1.4 \mathrm{GHz}$ and the maximum return loss is $-34 \mathrm{~dB}$. The microstrip patch antenna has 3D radiation pattern at $5.89 \mathrm{GHz}$ as shown in Figure 4 (b), and the gain of antenna is $5.25 \mathrm{~dB}$. Figure 4 (d) shows the half power beam width (HPBW) of the microstrip patch antenna, and the HPBW of antenna is $95^{\circ}$.

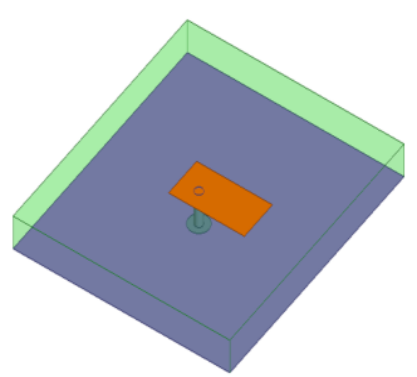

$4(a)$

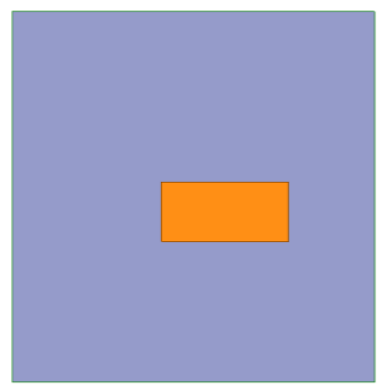

4(b)

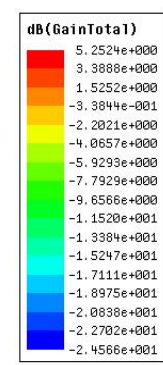

$-2.2702 e+001$
$-2.4566 e+001$

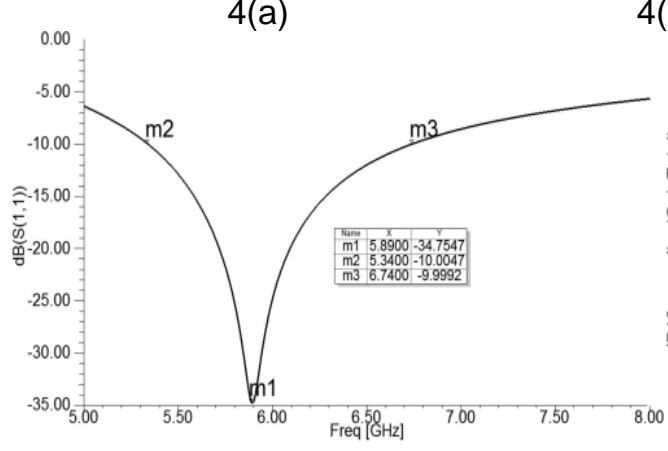

$4(d)$

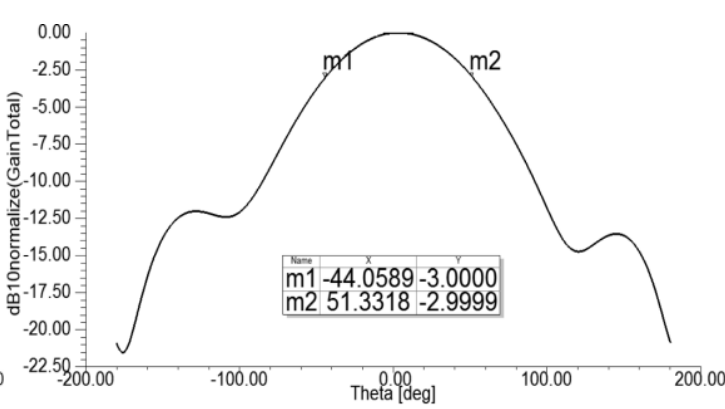

$4(e)$

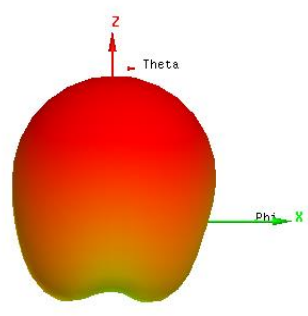

$4(c)$

Figure 4. The Performance of the Microstrip Patch Antenna: (a) Side View; (b) Front View, (c) Gain, (d) Return Loss, (e) HPBW 


\section{High Gain Microstrip Antenna with Left-Handed Material}

A lattice of the forementioned novel S-shaped material was constructed to form a $1 \times 23$ array and located inside the FR4 slab. The radome is consist of the slab and the $\mathrm{S}$-shaped material array, as shown in Figure 5 (a).

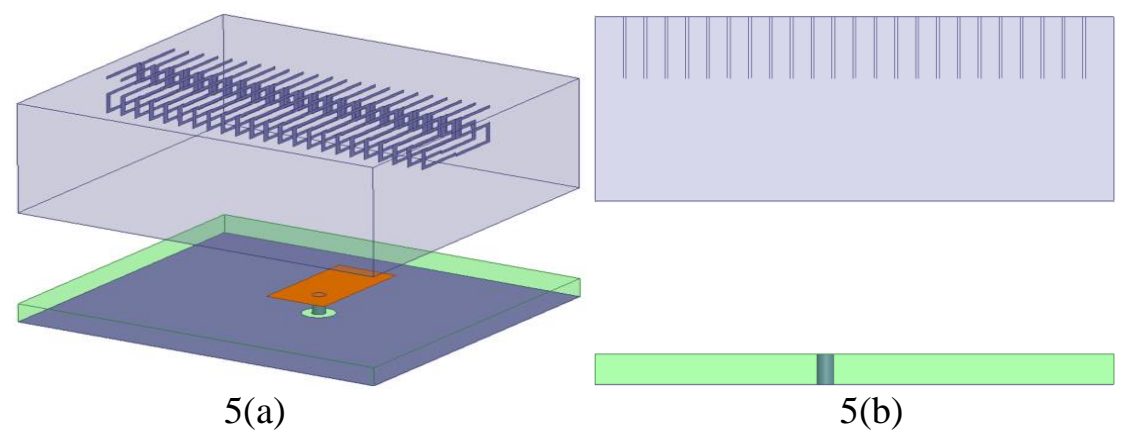

Figure 5. Sketch Graph of the Antenna System: (a) Front view, (b) Side-View

In order to make the front and back reflection waves counteract, the electrical thickness of the radome should be equal to the half of the wavelength in the medium. In this case, the size of the radome is $36 \times 32 \mathrm{~mm}^{2}$, the height of the radome is $12 \mathrm{~mm}$, and the $\mathrm{S}$-shaped materials arrays are inserted at the top of the radome. The distance between the radome and microstrip patch antenna is optimized to $10 \mathrm{~mm}$.

Figure 6(a) shows the return loss of the antenna system. It can be observed that the bandwidth of the antenna system is $1.64 \mathrm{GHz}$ and the maximum return loss is $-30 \mathrm{~dB}$. Figure 6(c) illustrates the gain of antenna system is $9.19 \mathrm{~dB}$. The HPBW of antenna system is $56.1^{\circ}$, as shown in Figure 6(b).
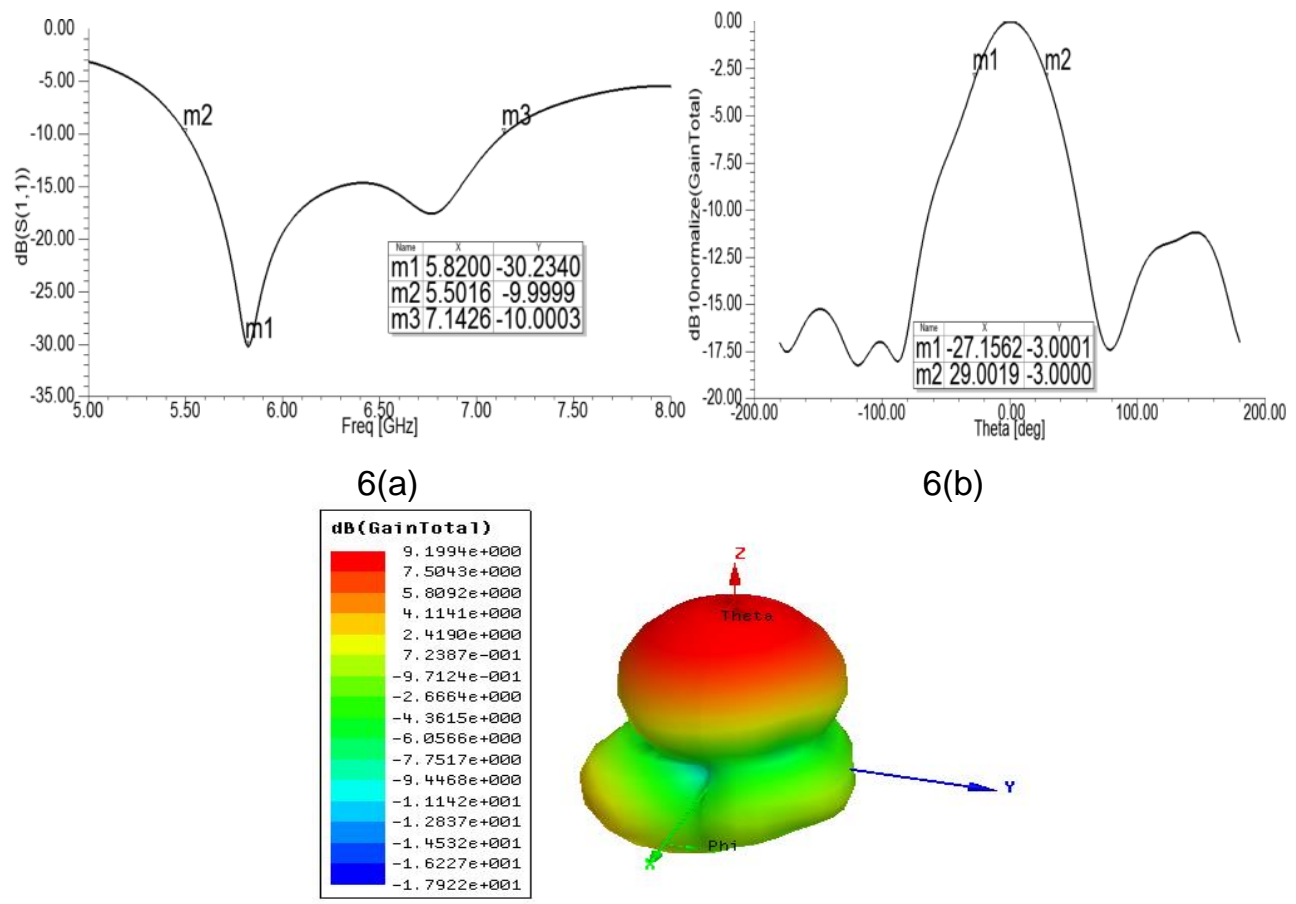

$6(b)$

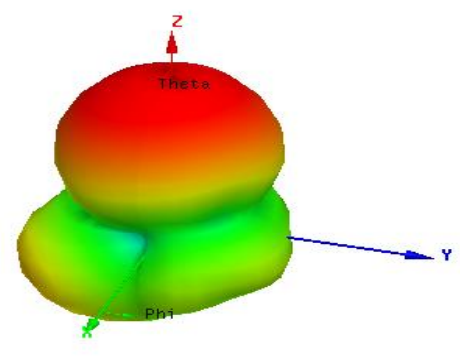

6(c)

Figure 6. The Performance of the Antenna System: (a) Return Loss, (b) HPBW, (C) Gain 
Table 1 is constructed to illustrate the difference performance between the microstrip patch antenna and the antenna system. It is obvious that the return loss, gain, HPBW are improved with slightly shift of the operating frequency.

Table 1. The Difference Performance Between the Microstrip Antenna and the Antenna System

\begin{tabular}{ccc}
\hline performance & microstrip antenna & antenna system \\
\hline operating frequency & $5.89 \mathrm{GHz}$ & $5.82 \mathrm{GHz}$ \\
bandwidth & $1.4 \mathrm{GHz}$ & $1.64 \mathrm{GHz}$ \\
gain & $5.25 \mathrm{~dB}$ & $9.19 \mathrm{~dB}$ \\
HPBW & $95^{\circ}$ & $56.1^{\circ}$ \\
\hline
\end{tabular}

\section{Conclusion}

In this paper, a novel S-shaped left-handed material is proposed. In order to have an accurate comparison, a conventional microstrip antenna is designed at first. Then a high gain microstrip antenna is designed with the radome consisted of the novel S-shaped materials. The performance of the microstrip antenna is compared with the performance of the modified antenna system. The simulation results show that the conventional microstrip antenna has a bandwidth of $1.4 \mathrm{GHz}$, and the gain of the microstrip antenna is $5.25 \mathrm{~dB}$, the HPBW is $95^{\circ}$. The simulation results also show that the bandwidth of the modified antenna system is $1.64 \mathrm{GHz}$, and the gain of the antenna system is $9.19 \mathrm{~dB}$, the $\mathrm{HPBW}$ is $56.1^{\circ}$. Therefore, there is an improvement in the antenna characteristics in term of gain, bandwidth, and HPBW. The gain of the microstrip antenna increased by $3.94 \mathrm{~dB}$ with left-handed material, as well as the HPBW decreased by $38.9^{\circ}$. The bandwidth of antenna improved from $1.4 \mathrm{GHz}$ to $1.64 \mathrm{GHz}$, with slightly shift of the operating frequency. It is clear that the performance of microstrip antenna can be improved by using left-handed material.

\section{References}

[1] D.R. Smith, D.C. Vier, N. Kroll and S. Schultz, "Direct calculation of permeability and permittivity for a left-handed metamaterial", Application on Physical Letters, vol. 77, no. 14, (2000).

[2] D. R. Smith and N. Kroll, "Negative refractive index in left-handed materials", Phys. Rev. Lett., vol. 85, no. 14, (2000).

[3] S. K. Patel and Y. P. Kosta, "Multiband metamaterial truncated square microstrip-based radiating structure design", Waves Random Complex Media,. vol. 24, no. 1, (2014).

[4] Y. Shen and L. L. Gong, "Investigation of gain effect of multi-band patch antenna based on composite rectangular”, SRRs. Optik. vol. 125, (2014).

[5] M.K.A. Rahim, N. Ibrahim, H.A. Majid and N.A. Murad, "Left-handed metamaterial structure incorporated with microstrip antenna", Microw. Opt Techn. Lett., vol. 54, no. 12, (2012).

[6] A.C.D. Souza, T.P. Vuong and C. Defay, "High gain circular polarization antenna for $5.8 \mathrm{GHz}$ with left-handed aaterials", European Conference on Antennas and Propagation, (2011).

[7] S. L. Hou, S. J. Zhang, Y. J. Liu and D. B. Wang, "Investigation on characteristics of W-type fiber with an inner cladding made of negative refractive index materials", Optik, vol. 125, (2014).

[8] C. P. Wang, "A rapid auto-focusmethod in the telephoto lens. Review of computer engineering studies", vol. 2, no. 2, (2015)

[9] Y. Zhao, C. Y. Feng, J. Yang and L. M. Wang, "Literature review of network public opinion about the e-commerce. Review of computer engineering studies", vol. 2, no. 2, (2015).

[10] C. Wang and S.M. Zhu, "A design of FPGA-based system for image processing. Review of computer engineering studies", vol. 2, no. 1, (2015).

[11] G.S. Rajput "Design and analysis of rectangular microstrip patch antenna using metamaterial for better efficiency. International Journal of Advanced Technology \& Engineering Research", vol. 2, no. 6, (2012).

[12] A. Sharma, S.K. Gupta, B.K. Kanaujia, G. P. Pandey and M. Sharma, "Design and analysis of a meandered multiband antenna based on split ring resonator", Microw. Opt Techn. Lett., vol. 55, no. 11, (2013). 
[13] A. Belenguer, J. Cascon, A. L. Borja, H. Esteban and V. E. Boria, "Dual composite right-/left-handed coplanar waveguide transmission line using inductively connected split-ring resonators", IEEE Trans. Microw. Theory Tech., vol. 60, no. 10, (2012).

[14] K. S. Zheng, N. J. Li, A. K. Ren, G. Wei and J. D. Xu, "Designing and measurement of a single layered planar gain-enhanced antenna radome with metamaterials", J. of Electromagn. Waves and Appl., vol. 26, (2012).

[15] P. Katiyar and W. N. L. W. Mahadi, "Impact analysis on distance variation between patch antenna and metamaterial”, Microw. Opt Techn. Lett., vol. 57, no. 1, (2015).

[16] M. F. Khan, M. J. Mughal and M. Bilal, "Rotation-a technique to tune the working frequency of left-handed materials", Microw. Opt Techn. Lett., vol. 53, no. 11, (2011).

[17] H. S. Chen, L. X. Ran, J. T. Huangfu, X. M. Zhang and K. S. Chen, "Left-handed materials composed of only S-shaped resonators", Rhys. Rev. E, vol. 70, (2004).

[18] M. F. Khan, M. J. Mughal and M. Bilal, "Effective permeability of an s-shaped resonator", Microw. Opt Techn. Lett., vol. 54, no. 2, (2012).

[19] D. R. Smith, W. J. Padilla, D. C. Vier, S. C. Nemat-Nasser and S. Schultz, "Composite medium with simultaneously negative permeability and permittivity", Phys. Rev. Lett., vol. 84, no. 18, (2000).

[20] H. S. Chen, L. X. Ran, J. T. huangfu, X. M. Zhang, K. S. Chen, T. M. Grzegorczyk and J. A. Kong, "Negative refraction of a combined double S-shaped metamaterial", Appl. Phys. Lett., vol. 86, (2005).

[21] J. B. Pendry, A. J. Holden, D. J. Robbins and W. J. Stewart, "Magnetism from conductors and enhanced nonlinear phenomena", IEEE Transactions Microw, Theory Tech., vol. 47, no. 11, (1999). 
International Journal of Future Generation Communication and Networking Vol. 9, No. 3 (2016) 